\title{
To a man with a hammer, the world is a nail
}

\author{
John Matthew Toole, MD
}

See related article on pages 1312-21.

In their article in this issue of the Journal, Kukulski and colleagues $^{1}$ examine the results of surgical ventricular reconstruction (SVR) in patients with severe right ventricular (RV) dysfunction undergoing coronary artery bypass grafting (CABG) for ischemic cardiomyopathy in the Surgical Treatment for Ischemic Heart Failure (STICH) trial. Results of the surgical arm of the STICH trial ${ }^{2}$ have been controversial and disappointing to some. Numerous post hoc analyses have been performed in an effort to glean more useful information from this herculean surgical randomized clinical trial. In this post hoc analysis, Kukulski and colleagues ${ }^{1}$ attempt to do just that.

Despite the recruitment and randomization of 1000 surgical patients, no differences in clinical outcomes were observed in those undergoing SVR in addition to CABG in the STICH trial. Proponents of SVR claim that the exclusion criteria were too liberal and that reduction in end-systolic volume index was inadequate to accurately assess the benefits of SVR. Others believed that the trial was acceptable because SVR was examined under the real-life conditions in which indications for this procedure had evolved. In response to the STICH trial, Dor and colleagues $^{3}$ reported a retrospective series of 267 patients meeting exclusion criteria for the STICH trial who had excellent clinical results after SVR. In this series, Dor and colleagues $^{3}$ observed a $50 \%$ reduction in end-systolic volume index versus $19 \%$ in the STICH trial. This raised the question of whether SVR was inappropriately used or overused in the STICH trial. In a separate post hoc analysis of the STICH trial, Michler and colleagues ${ }^{4}$ found a survival benefit for those in whom an end-systolic volume index of $70 \mathrm{~mL} / \mathrm{m}^{2}$ or less was achieved relative to those undergoing CABG alone. This suggested that SVR might be beneficial to those with a larger degree of ventricular remodeling. Holly and colleagues ${ }^{5}$ examined the effect of preoperative myocardial viability on surgical outcomes. Intuitively, it

From the Division of Cardiothoracic Surgery, Medical University of South Carolina, Charleston, SC.

Disclosures: Author has nothing to disclose with regard to commercial support.

Received for publication Jan 5, 2015; accepted for publication Jan 5, 2015; available ahead of print Feb 7, 2015.

Address for reprints: John Matthew Toole, MD, Medical University of South Carolina, Cardiothoracic Surgery, 25 Courtenay Dr, ART 7026, Charleston, SC 29425 (E-mail: toole@musc.edu).

J Thorac Cardiovasc Surg 2015;149:1322-3

$0022-5223 / \$ 36.00$

Copyright (c) 2015 by The American Association for Thoracic Surgery

http://dx.doi.org/10.1016/j.jtcvs.2015.01.011 seems that exclusion of scar might be more beneficial than exclusion of hibernating myocardium; however, no difference in outcome based on viability was identified. On the basis of the results of Dor and colleagues ${ }^{3}$ and Michler and colleagues, ${ }^{4}$ there is evidence that SVR does benefit some patients. Indications and, perhaps more importantly, contraindications to SVR still need to be elucidated clearly.

In their article, Kukulski and colleagues ${ }^{1}$ were able to demonstrate a difference in the combined end point of death or readmission for heart failure and the secondary end point of all-cause mortality according to the presence of moderate to severe RV dysfunction. They found a hazard ratio of 1.86 for the primary end point of all-cause mortality or cardiovascular hospital readmission and a hazard ratio of 3.37 for the secondary end point of all-cause mortality. Both these findings were statistically significant. This is useful information but somewhat intuitively obvious. RV dysfunction is a product of pulmonary hypertension. In the setting of ischemic cardiomyopathy, pulmonary hypertension is probably due to elevated left ventricular (LV) end-diastolic pressure and is simply a sign of advanced and long-standing LV dysfunction. The prognostic value of LV dysfunction in cardiac surgery is well established, so these findings are not surprising. The improvements in myocardial function resulting from concomitant CABG at the time of SVR are not predictable. Severe RV dysfunction may indicate that these improvements are unlikely to occur, at least to any physiologically meaningful degree.

There are limitations to this study that need be considered. This is a post hoc analysis that analyzes a variable not taken into account during randomization in the STICH trial. Although the study is of higher quality than a retrospective review, the results must be interpreted with some caution because clinical bias cannot be excluded. The largest weakness of this study is the small number of patients noted to have RV dysfunction. Of the 1000 patients randomly assigned in the STICH trial, only 78 were found to have moderate or severe RV dysfunction. This study examined RV function on the basis of echocardiographic data alone, which is a highly subjective and inexact method. This is partially balanced out by the use of a blinded core echocardiographic laboratory. Theoretically, subjectivity should be consistent, with the bias minimized by blinding.

The role of SVR in the toolbox of the surgeon treating heart failure still remains unclear. The theory behind SVR is that reduction of LV diameter causes an exponential reduction in wall tension. Wall tension is physiologically 
equivalent to oxygen demand. This theory is consistent with the findings of Dor and colleagues ${ }^{3}$ and Michler and colleagues, ${ }^{4}$ in which larger reductions in LV size were observed. Broad application of SVR with modest reduction in end-systolic volume index, as observed in the STICH trial, is not beneficial. Likewise, it is important to discriminate patients who are likely to experience a detrimental effect from SVR and use a more appropriate tool to treat their heart failure. Such tools include medical management, isolated CABG, transplant, and LV assist device implantation.

\section{References}

1. Kukulski T, She L, Racine N, Gradinac S, Panza J, Velazquez E, et al. Implications of right ventricular dysfunction on long-term outcome in patients with ischemic cardiomyopathy undergoing coronary artery bypass grafting with or without surgical ventricular reconstruction. J Thorac Cardiovasc Surg. 2015;149:1312-21.

2. Jones RH, Velazquez EJ, Michler RE, Sopko G, Oh JK, O'Conner CM, et al; STICH Hypothesis 2 Investigators. Coronary artery bypass surgery with or without surgical ventricular reconstruction. N Engl J Med. 2009;360:1705-17.

3. Dor V, Civaia F, Alexandrescu C, Sabatier M, Montiglio F. Favorable effects of left ventricular reconstruction in patients excluded from the Surgical Treatment for Ischemic Heart Failure (STICH) Trial. J Thorac Cardiovasc Surg. 2011;141: 905-16, 916.e1-4.

4. Michler RE, Rouleau JL, Al-Khalidi HR, Bonow RO, Pellikka PA, Pohost GM, et al; STICH Trial Investigators. Insights from the STICH trial: change in left ventricular size after coronary artery bypass grafting with and without surgical ventricular reconstruction. J Thorac Cardiovasc Surg. 2013;146:1139-45.e6.

5. Holly TA, Bonow RO, Arnold JM, Oh JK, Varadarajan P, Pohost GM, et al Myocardial viability and impact of surgical ventricular reconstruction on outcomes of patients with severe left ventricular dysfunction undergoing coronary artery bypass surgery: results of the Surgical Treatment for Ischemic Heart Failure trial. J Thorac Cardiovasc Surg. 2014;148:2677-84.e1. 